\title{
Dynamics, Control, and Modeling of Fractional-Order Systems
}

\author{
José M. Balthazar $\mathbb{D}^{1},{ }^{1}$ Paulo B. Gonçalves $\mathbb{D},{ }^{2}$ Grzegorz Litak (D), \\ Angelo $M$. Tusset $\left(\mathbb{B},{ }^{4}\right.$ and Livija Cveticanin $(\mathbb{D})^{5}$ \\ ${ }^{1}$ Aeronautics Technological Institute (ITA), São José dos Campos, SP, Brazil \\ ${ }^{2}$ Pontifical Catholic University of Rio de Janeiro (PUC-Rio), Rio de Janeiro, Brazil \\ ${ }^{3}$ Lublin University of Technology, Lublin, Poland \\ ${ }^{4}$ Federal University of Technology-Paraná (UTFPR), Ponta Grossa, PR, Brazil \\ ${ }^{5}$ University of Novi Sad, Novi Sad, Serbia \\ Correspondence should be addressed to José M. Balthazar; jmbaltha@gmail.com
}

Received 14 May 2018; Accepted 15 May 2018; Published 31 July 2018

Copyright ( $(2018$ José M. Balthazar et al. This is an open access article distributed under the Creative Commons Attribution License, which permits unrestricted use, distribution, and reproduction in any medium, provided the original work is properly cited.

This special issue addresses nonlinear system models considering the dynamical analysis, control approaches, and applications in the fractional-order case and their applications to engineering. It involves modeling, applications, and control for nonlinear systems, such as mechanical and electromechanical ones. Five relevant contributions from researchers in different topics of nonlinear systems, describing original theoretical research as well as new experimental results, were published in the presented special issue (SI). The five contributions are summarized, next.

K. Rajagopal et al. analyzed the chaotic behavior using bifurcation diagrams of horizontal platform systems (HPS) identifying the multistable parameter and investigated the coexisting attractors of the HPS. The authors in the work titled "Multistability in Horizontal Platform System with and without Time Delays" investigated the multistability existence in time-delayed HPS, plotting the bifurcation of the autonomous HPS, and showed the multistability and coexisting attractors. D. G. Bassinello et al. studied a nonlinear MEMS resonator, bringing the behavior of such system from a chaotic state to a periodic orbit, considering the timedelayed feedback control, and the sliding mode control, in the paper entitled "Dynamical Analysis and Control of a Chaotic Microelectromechanical Resonator Model". J. Wang et al. discussed in detail the dynamical characteristics of a blade with viscoelastic damping in the paper entitled "Dynamic Characteristics of Blade with Viscoelastic Damping Block
Based on Complex Eigenvalue Method". The effects of various parameters including thickness, storage modulus, loss factor of viscoelastic damping block, and rotating speed on natural frequency and modal damping ratio of VE-blade were considered. M.-H. Shih et al. considered the Bi-Tilt Isolator (BTI) composed of bi-tilt beveled substrate and slider in the paper "Experimental Validation of Numerical Model for BiTilt-Isolator". The proposed mathematical model and BTI element of GENDYN program were analyzed, using cubic polynomial function of friction. The authors carried out fine simulation capability to assess the nonlinear isolation effect of the structure installed with BTI. T. Qin et al. have performed a series of tests on characteristics of acoustic emission sandstone under uniaxial, conventional, triaxial conditions and the unloading confining pressure path. They observed in the paper with title "Experimental Study on Mechanical and Acoustic Emission Characteristics of Rock Samples under Different Stress Paths" that the failure mode of rock specimen was dominated by shear failure under the conventional triaxial stress path and the tension failure was the main form at a lower initial value of unloading confining pressure. In addition, they showed that the shear failure is more prominent at a higher initial value of unloading confining pressure.

We hope that this special issue would be useful in recent advances and developments in the areas of Dynamics, Control, and Modeling of Fractional-Order Systems and 
attract attention of the scientific community to pursue further research and further studies.

\section{Acknowledgments}

We would like to acknowledge all the authors for their informative contributions and the reviewers for their support and constructive critiques in making this special issue possible.

José M. Balthazar Paulo B. Gonçalves Grzegorz Litak Angelo M. Tusset Livija Cveticanin 


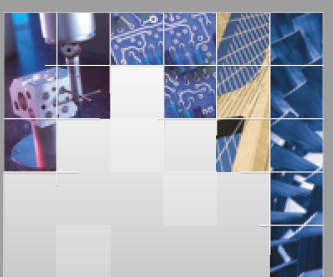

\section{Enfincering}
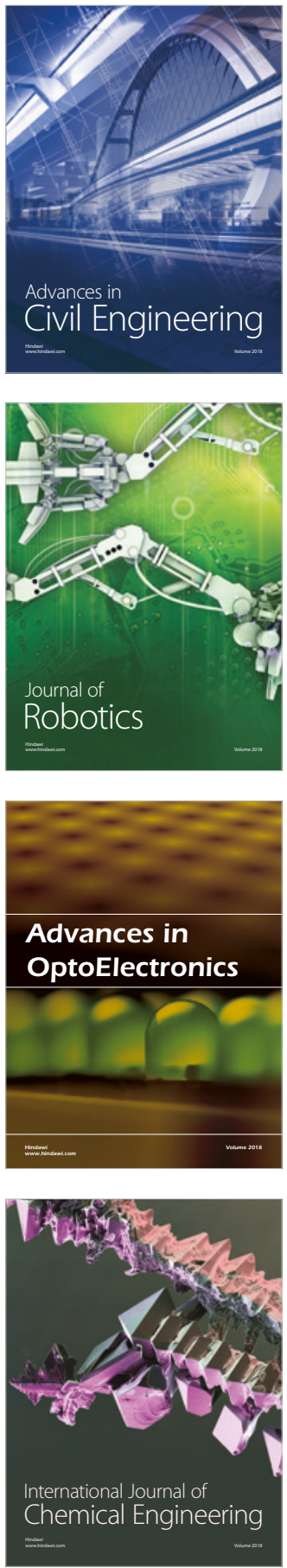

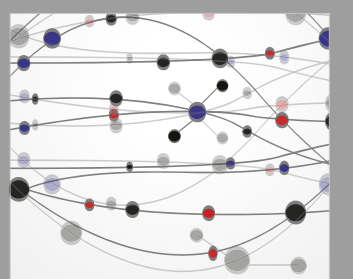

\section{Rotating \\ Machinery}

The Scientific World Journal

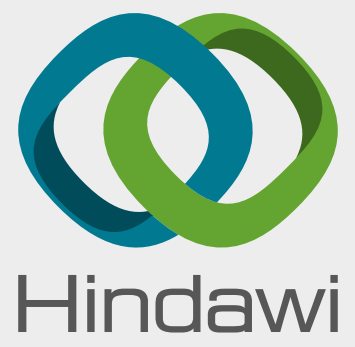

Submit your manuscripts at

www.hindawi.com
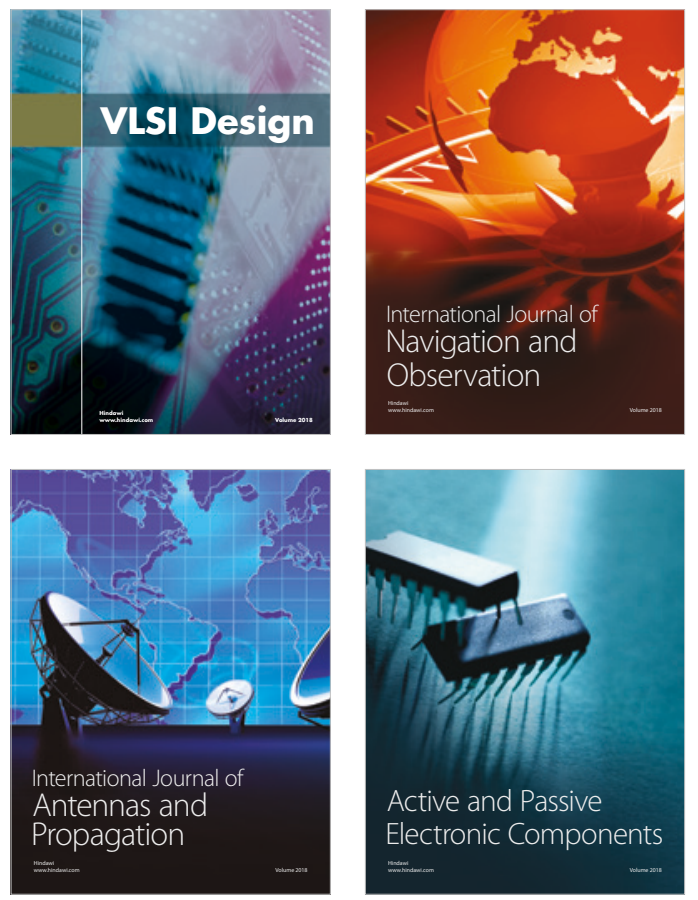
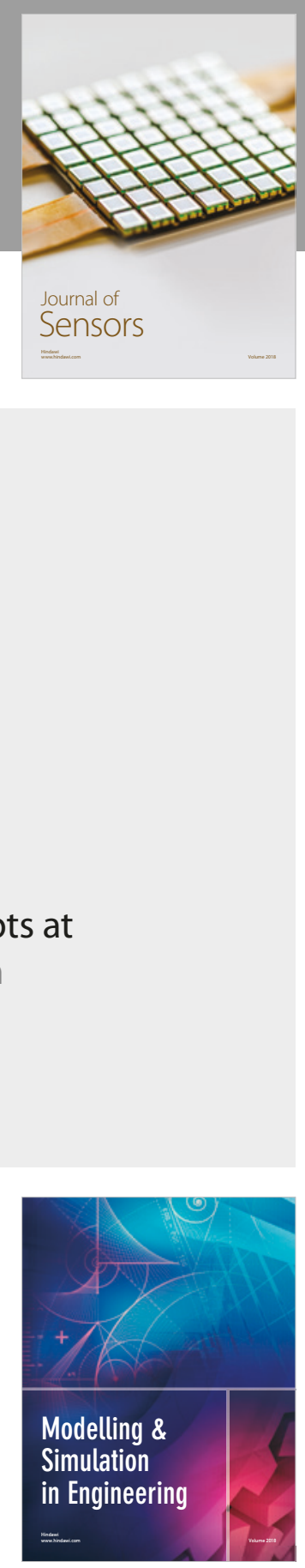

\section{Advances \\ Multimedia}
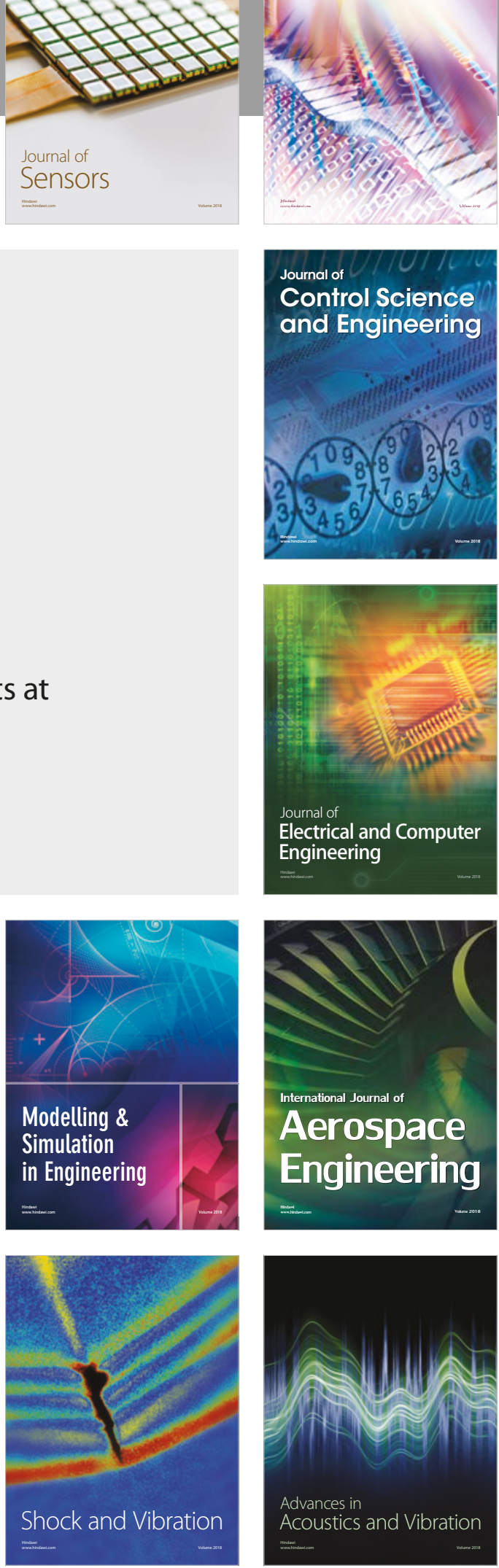